\title{
LE TOURNEAU François-Michel, Les Yanomami du Brésil. Géographie d'un territoire amérindien
}

\section{Xavier Arnauld de Sartre}

\section{OpenEdition}

\section{Journals}

Édition électronique

URL : http://journals.openedition.org/jsa/11788

DOI : 10.4000/jsa.11788

ISSN : 1957-7842

\section{Éditeur}

Société des américanistes

\section{Édition imprimée}

Date de publication : 5 octobre 2011

Pagination : 358-363

ISSN : 0037-9174

\section{Référence électronique}

Xavier Arnauld de Sartre, «Le tourneau François-Michel, Les Yanomami du Brésil. Géographie d'un territoire amérindien », Journal de la Société des américanistes [En ligne], 97-1 | 2011, mis en ligne le 10 juillet 2011, consulté le 21 septembre 2020. URL : http://journals.openedition.org/jsa/11788 ; DOI : https://doi.org/10.4000/jsa.11788

Ce document a été généré automatiquement le 21 septembre 2020

(c) Société des Américanistes 


\title{
LE TOURNEAU François-Michel, Les Yanomami du Brésil. Géographie d'un territoire amérindien
}

\author{
Xavier Arnauld de Sartre
}

\section{RÉFÉRENCE}

LE TOURNEAU François-Michel, Les Yanomami du Brésil. Géographie d'un territoire amérindien, Belin, coll. « Mappemonde », Paris, 2010, 479 p., bibl., ill., cartes, photos, tabl.

1 Hasard éditorial... et hasard du calendrier des recensions : l'ouvrage de François-Michel Le Tourneau sur le territoire Yanomami paraît (presque) au même moment que l'autobiographie à deux voix de Davi Kopenawa et Bruce Albert, et il est donc heureux que ce compte rendu accompagne celui de José Kelly sur La chute du ciel. Paroles d'un chaman yanomami. Le hasard fait bien les choses, tant les deux ouvrages se complètent, l'un (celui de François-Michel Le Tourneau) décrivant, comprenant et parfois condamnant, sans l'avoir prémédité, la face blanche du processus décrit de l'intérieur par l'autre, en même temps qu'il propose une lecture globale du territoire yanomami. La complémentarité des deux ouvrages peut se lire à trois niveaux au moins : au niveau des croisements entre les itinéraires des auteurs de chacun des ouvrages, au niveau, nous venons de le dire, de la lecture croisée du même phénomène, enfin, au niveau de la complémentarité entre les disciplines de chacun des auteurs, l'anthropologie et la géographie.

2 Le premier niveau paraitt le plus anecdotique, mais il n'en est pas moins utile. Au fil des pages de l'ouvrage de François-Michel Le Tourneau, qui décrit à peu de choses près la même période historique que celle couverte par la vie de Davi Kopenawa, nous voyons agir celui-ci et Bruce Albert, qui sont ainsi resitués dans leur contexte historicogéographique. Bruce Albert est le premier à apparaître, sa figure se dessinant au fur et à mesure que la lutte pour la reconnaissance des droits yanomami s'installe et se 
résout, au moins temporairement: si son itinéraire est intimement lié à celui de la reconnaissance des Yanomami, c'est que Bruce Albert a joué un rôle fondamental dans cette reconnaissance. Le jeune anthropologue qu'il est dans les années 1980 participe au premier projet affichant explicitement son objectif de reconnaissance des Yanomami : le projet Yanoama de Kenneth Taylor. Sa figure se précise au travers des analyses de la création et du fonctionnement de l'ONG CCPY, sans laquelle les Yanomami ne seraient pas ce qu'ils ont réussi à devenir aujourd'hui. Il est toujours présent quand, dans les troisième et quatrième parties de l'ouvrage, est posée la question de la structuration et du futur du territoire yanomami. Il faut dire que Bruce Albert a joué un rôle essentiel dans l'entreprise de François-Michel Le Tourneau, puisque c'est lui qui l'a pour la première fois invité chez les Yanomami et qui a en partie guidé ses pas tout au long de ces années passées à travailler avec et sur ces Indiens. Quant à Davi Kopenawa, son rôle dans la structuration et la résonnance internationale de ce territoire est aussi clairement mis en évidence, en particulier dans les dernières parties de l'ouvrage où de longs développements lui sont consacrés.

Cependant, l'ouvrage de François-Michel Le Tourneau constitue bien évidemment plus qu'une simple mise en contexte des deux auteurs de l'ouvrage au regard duquel nous le lisons. Il en constitue la face blanche - au sens où il peut apparaître comme l'étude fine des logiques qui, du côté des Blancs, ont présidé au contact des Yanomami, à leur tentative d'ethnocide, puis à la reconnaissance de leurs droits. Le scientifique le sait, mais il n'est jamais inutile de le rappeler : étude compréhensive (des logiques de la face blanche) du contact avec les Yanomami ne veut pas dire blanchiment des conséquences que ce contact a eues. Bien au contraire, François-Michel Le Tourneau démontre, archives à l'appui, que les différents pouvoirs qui se sont succédé avaient une conscience claire des impacts qu'auraient sur les Indiens les actions qu'ils ont laissé se dérouler. Il décrit l'ethnocide presque achevé des Yanomami en en montrant à la fois les logiques et la non-nécessité, le fait qu'il aurait pu être évité si les rapports de force interne au Brésil, comme on l'imagine aisément pour toute autre puissance colonisatrice, avaient été différents. Ce faisant, il permet d'imaginer très clairement le processus selon lequel d'autres ethnocides sont, eux, arrivés à terme.

4 Le fait que François-Michel Le Tourneau ait été au contact, presque étroit, des acteurs de la défense des Indiens n'impliquait pas nécessairement que son argumentaire prenne cette forme. Ce serait méconnaître à la fois les conditions dans lesquelles il s'est engagé dans son étude des Yanomami et l'objectivité dont il a constamment voulu faire preuve dans son analyse. Le premier "contact» avec Bruce Albert a été rugueux, et François-Michel Le Tourneau n'était pas prédestiné à devenir un défenseur de la cause indigène. Sa justesse s'est petit à petit imposée à lui. Quand l'auteur affirme, dans son avant-propos, qu'il pense que "[sa] vision" n'a pas été plus «brouillée » que son « traitement de l'information » n'a été « rendu partisan » par sa proximité des ONG, on peut lui faire confiance : il se fonde, pour son étude du contact entre les Yanomami et le reste du monde, sur des archives de ce contact, archives qu'il replace dans leurs contextes historiques. À plusieurs reprises, lorsqu'il manque d'archives, lorsque les discussions semblent s'être déroulées dans des coulisses dont il ne reste nulle trace, François-Michel Le Tourneau est conduit à émettre des hypothèses qui emportent d'autant plus l'adhésion du lecteur que leur bien-fondé est démontré à la fois par les logiques générales des différents acteurs incriminés et par les conséquences avérées de telles actions. À aucun moment l'auteur ne peut être pris à défaut dans son 
argumentation... ce qui rend d'autant plus implacable sa dénonciation, involontaire pourrait-on dire, de ce contact pour le moins problématique.

De ce fait, Le Tourneau est un défenseur non militant, objectif pourrait-on dire, des Yanomami. Est-il nécessaire de rappeler combien ce type d'argumentaire est nécessaire ? Il apparaissait évident que l'ouvrage du duo Kopenawa-Albert pourrait être lu comme un plaidoyer pour la défense des Yanomami, Bruce Albert endossant ce rôle dès le début du livre. En revanche, les sceptiques - et les spécialistes de la fabrication de l'opinion savent qu'ils sont nombreux - ne pourront pas, à la lecture de l'ouvrage de François-Michel Le Tourneau, attribuer l'analyse à la partialité de l'auteur.

6 Mais foin de politique. Ce serait trahir le projet de François-Michel Le Tourneau que de lui accorder plus de place qu'elle n'en a eu dans la démarche de l'auteur. Ce serait masquer les très belles pages qui supportent son propos. En effet, François-Michel Le Tourneau n'est pas qu'« objectif ", c'est aussi un auteur accompli. Il a pris un plaisir évident à écrire ce livre. Le plaisir est contagieux, bien évidemment, et le lecteur se plaît à suivre l'auteur dans les détails qu'il donne de la construction d'une route en forêt amazonienne, du fonctionnement du garimpagem, des scènes politiques et de leurs coulisses, de l'entreprise pionnière ou du fonctionnement d'un «territoire indigène ». Tant d'univers différents, plus opposés qu'alliés, participent de la construction et de l'avenir de ce territoire, que l'on est conquis par le tour de force qui a consisté à les réunir en un seul ouvrage.

7 C'est là l'entreprise d'un géographe dont l'objectif fut de montrer comment se construit un territoire, une région. Ce projet, qui est celui de la géographie régionale à laquelle François-Michel Le Tourneau se réfère dans l'introduction de l'ouvrage, prend un tout autre sens dès lors qu'il s'agit d'un territoire indigène. Jusqu'à présent, lorsque l'on étudiait la formation d'une région en Amazonie, c'était par le biais de l'entreprise pionnière. Olivier Dollfus et Pierre Monbeig ont les premiers montré la voie, et Le Tourneau est de ce point de vue leur digne héritier. Toutefois, une démarche nettement moins habituelle en géographie est requise pour lire un processus de régionalisation dans le cas de sociétés totalement étrangères non seulement à l'idée de région, mais plus encore, à leur propre identité collective - étrangeté à laquelle fait écho la négation de ces populations par un grand nombre d'acteurs ayant une influence essentielle sur leur devenir.

8 La première partie de l'ouvrage est consacrée à la manière dont ce peuple s'est révélé à lui-même en même temps qu'il s'est confronté à l'altérité radicale, à l'homme blanc. Jusqu'au contact prolongé avec les Blancs, François-Michel Le Tourneau explique que les Yanomami étaient un peuple pour qui la figure de l'autre était incarnée par les groupes indigènes qui n'étaient ni leurs amis ni leurs ennemis, et qui leur étaient de ce fait vaguement hostiles. C'est dire le choc que constitua pour eux le contact prolongé avec les Blancs, puis la prise de conscience du monde dont ces derniers ne sont en fait que l'avant-garde. C'est dire aussi l'ampleur du travail sur eux-mêmes qui leur fut nécessaire pour se constituer en tant que société. Le choc fut certes moins violent pour les Blancs, mais ceux-ci n'en eurent pas moins du mal à reconnaître les territorialités indigènes - pour des raisons tant conceptuelles que, l'on s'en doute, politicoéconomiques.

9 C'est ce double processus de reconnaissance que François-Michel Le Tourneau décrit dans la deuxième partie de son ouvrage. Celle-ci s'ouvre sur une mise en évidence de l'univers des possibles en termes de reconnaissance du territoire yanomami, 
possibilités qui se structurent autour d'une opposition radicale : faut-il reconnaître un seul territoire de grande taille, ou se contenter de démarquer de multiples îlots? Qu'est-ce qui préside à ces choix ? Sur quelles connaissances de la réalité ces choix sont-ils fondés? Quels acteurs agissent en faveur de l'un ou de l'autre ? Quelles logiques ces acteurs suivent-ils? Voire pour certains d'entre eux : quels intérêts les animent? Quels conflits l'hésitation entre ces deux possibilités révèle-t-elle? L'analyse est ici très fine, et Le Tourneau montre bien que les hésitations des Blancs sont dans un premier temps le fait de leur relative ignorance de la situation des Yanomami, puis celle de leur volonté délibérée d'ignorer cette situation.

10 La complexité des Blancs va bien évidemment au-delà d'une simple opposition entre les défenseurs de la cause indigéniste et les autres, sourdement hostiles aux Indiens quand ils ne les massacrent pas directement. Essayons de reprendre quelques-uns des principes qui différencient les différents protagonistes en groupes d'acteurs aux intérêts contradictoires. Certes, le choix de l'engagement au côté des Indiens est bien évidemment crucial pour comprendre les logiques d'action des indigénistes. Néanmoins, pour les autres acteurs, la périodisation politique est un facteur tout aussi essentiel que celui de la connaissance des Yanomami pour comprendre le sort qui leur est réservé : la dictature d'abord, le retour à la démocratie ensuite ${ }^{1}$, et le Brésil d'après Constitution de 1988 enfin... Le niveau d'échelle duquel ces acteurs tirent leur légitimité et où ils placent leurs actions est tout aussi important : le niveau fédéral est soit opposé, soit allié, avec le niveau étatique ; les garimpeiros tentent de faire adhérer à leur cause les politiques locaux - et la perte de leur soutien leur sera d'ailleurs (provisoirement?) fatale ; les indigénistes, alliés à certains missionnaires, tirent quant à eux profit de leur légitimité internationale pour renforcer leur action locale; d'autres acteurs, au contraire, tirent profit d'arguments nationalistes pour s'allier une opinion publique toujours encline à se méfier de l'intervention étrangère ${ }^{2}$. Des divisions plus subtiles encore apparaissent en outre au sein de chacun des niveaux : les différents services de l'Union, au premier rang desquels l'armée, ont des compétences et des instructions qui les conduisent à jouer des rôles différents; les Indiens eux-mêmes ne parlent pas tous d'une même voix, soit parce que le conflit guerrier structure profondément les relations des groupes entre eux, soit parce qu'au sein de chaque groupe les possibilités de troc avec les Blancs sont diversement appréciées.

11 Enfin, ces contradictions évoluent, des alliances se nouent et se dénouent... jusqu'au moment où, à la fin de la deuxième partie, peut être créé le territoire indigène. Création d'autant plus précieuse qu'elle n'était pas nécessaire: le vent du boulet ethnocidaire n'est pas passé loin des Yanomami. Dès lors, la troisième partie peut être consacrée à la fragile stabilisation de ce territoire au cours des années 1990 : alors que les plaies de l'expansion de la prospection minière ne sont pas encore pansées, il faut assurer le contrôle du territoire, mettre en place un système de santé efficace, et surtout, surtout, s'organiser. Ces trois processus ne se déroulent pas sans heurts et François-Michel Le Tourneau décrypte ce que les acteurs du développement ne savent que trop bien: une fois la survie d'une population assurée, une fois que l'action humanitaire a fait son œuvre, il faut organiser une population sans la nier, assurer non seulement sa survie, mais sa permanence identitaire dans un contexte qui a inexorablement changé, et inscrire sa propre action dans une temporalité forcément réduite... 

première heure doivent réinventer leur propre manière d'agir, ils doivent composer avec de nouveaux acteurs - alliés, certes, mais aux logiques d'actions différentes - et ils doivent accepter de transférer aux organes fédéraux, les ennemis d'hier, des compétences que ces derniers doivent assumer alors qu'ils ne sont pas toujours en mesure de le faire de manière efficace. Certes, cela se déroule dans un contexte globalement apaisé, mais l'on devine que cela ne va pas sans difficultés. À ce niveau, l'émergence d'un líder indigène tel que Davi Kopenawa est essentielle. C'est sur ces enjeux que se termine la troisième partie. Tourneau peut exercer son métier initial, celui de géographe. Ou, plus exactement, il peut étudier ce qui est souvent considéré comme le cœur du métier de géographe, l'organisation d'une portion d'espace et de ses relations avec d'autres portions d'espaces, situées à côté de ce lieu ou appréhendables à d'autres niveaux. La portion d'espace étudiée est, en l'occurrence, un territoire indigène. Si François-Michel Le Tourneau a su montrer que, pour comprendre la forme de ce territoire et les dynamiques qui l'animent, le géographe doit tour à tour se faire historien, analyste politique et décrypteur de l'action de développement, il sait aussi mobiliser les outils classiques de la géographie : analyse de la structuration de l'espace yanomami, de ses dynamiques démographiques, économiques et politiques et aussi de ses relations avec les autres niveaux scalaires, avec les régions environnantes et les fronts pionniers qui ne sont jamais loin.

$14 \mathrm{Au}$ terme de cette dernière partie, le sous-titre de l'ouvrage prend tout son sens: Géographie d'un territoire amazonien. C'est bien de cela qu'il s'agit : géographie régionale, certes, mais inaccessible sans un recours à l'histoire et sans une analyse des conflits culturels fondés sur des intérêts contradictoires... Une géographie qui, en outre, sait parfaitement utiliser tout le potentiel démonstratif des images, qui émaillent ce livre d'une très haute technicité : les cartes sont extrêmement précises, les illustrations nombreuses,... En somme, ce livre est bien celui d'un géographe qui a pleinement assumé les évolutions de sa discipline et les met remarquablement en pratique. Ces deux ouvrages qui renouvellent profondément la connaissance que nous avons des Yanomami illustrent la complémentarité des disciplines scientifiques auxquelles appartiennent François-Michel Le Tourneau et Bruce Albert, la géographie et l'anthropologie.

\section{NOTES}

1. À ce niveau, on notera que, contrairement à ce que l'on aurait pu croire, le retour de la démocratie a causé bien plus de mal aux Yanomami que la période de la dictature militaire.

2. François-Michel Le Tourneau analyse très finement le rôle non seulement d'acteurs non brésiliens dans la reconnaissance des territorialités yanomami, mais aussi les modalités selon 
lesquelles se construit un discours dénonçant une supposée internationalisation de l'Amazonie c'est d'ailleurs sur ce thème que se conclut l'ouvrage.

\section{AUTEURS}

\section{XAVIER ARNAULD DE SARTRE}

UMR Société environnement territoire, CNRS/Université de Pau et des Pays de l'Adour 